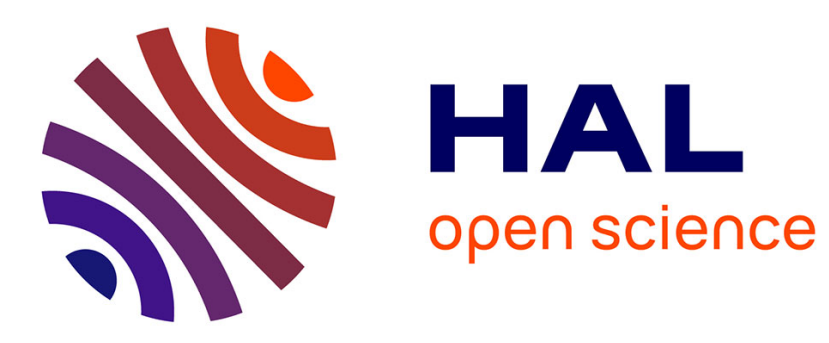

\title{
Cultes isiaques en Proconsulaire
}

Laurent Bricault, Yann Le Bohec, Jean-Louis Podvin

\section{To cite this version:}

Laurent Bricault, Yann Le Bohec, Jean-Louis Podvin. Cultes isiaques en Proconsulaire. IIe colloque international sur les études isiaques, 2002, Lyon, France. pp.221 - 241. hal-01817084

\section{HAL Id: hal-01817084 \\ https://hal.science/hal-01817084}

Submitted on 18 Jun 2018

HAL is a multi-disciplinary open access archive for the deposit and dissemination of scientific research documents, whether they are published or not. The documents may come from teaching and research institutions in France or abroad, or from public or private research centers.
L'archive ouverte pluridisciplinaire HAL, est destinée au dépôt et à la diffusion de documents scientifiques de niveau recherche, publiés ou non, émanant des établissements d'enseignement et de recherche français ou étrangers, des laboratoires publics ou privés. 


\section{Isis en Occident}

ACTES DU

II $^{\text {EMK }}$ COLLOQUE INTER NATIONAL

SUR LES ÉTUDES ISIAQUES,

LYON III, 16-17 MAI 2002

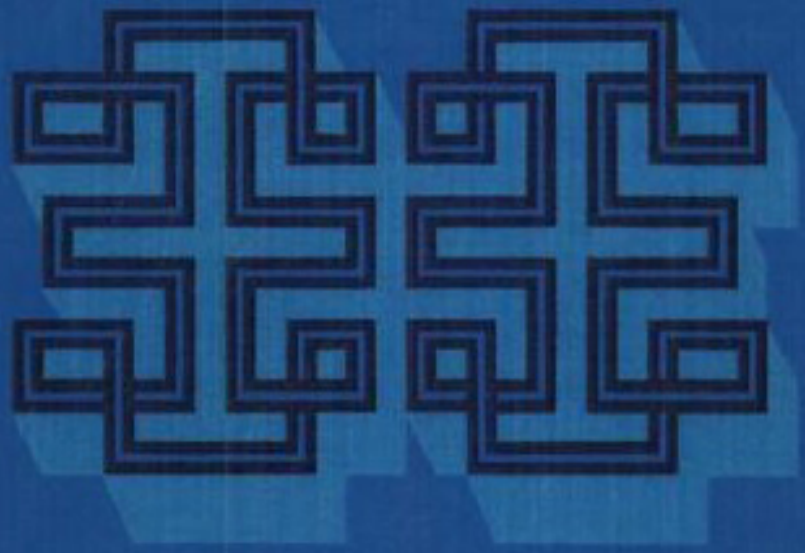

LAURENT BRICAULT

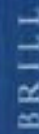




\section{CULTES ISIAQUES EN PROCONSULAIRE}

par

\section{Laurent Bricault, Yann Le Bohec et Jean-Louis Podvin}

Les plus anciennes traces de la présence d'Isis dans les régions qui deviennent à partir de 146 av. J.-C. la province romaine d'Africa se repèrent à Carthage. Les noms théophores égyptiens sont fort nombreux dans l'onomastique phénico-punique et parmi ceux-ci, les composés à partir du nom de la déesse, bien attestés dès le $\mathrm{VIII}^{\mathrm{e}}$ siècle av. J.-C. 'Isis est également présente dans l'iconographie phénicienne, comme dans l'épigraphie punique. ${ }^{2}$ La présence d'un culte rendu à Isis dans le monde phénico-punique doit pouvoir s'expliquer par le syncrétisme précoce qui l'identifia à Astarté, ${ }^{3}$ à Derkéto, ${ }^{4}$ puis à

' Cf. A. Lemaire, «Divinités égyptiennes dans l'onomastique phénicienne», dans C. Bonnet et al. (éds) Religio Phoenicia, Studia Phoenicia IV (Namur, 1986), pp. 87-98.

${ }^{2}$ On verra les références données par Corinne Bonnet, «Le culte d'Isis à Carthage. À propos de l'inscription funéraire punique CIS I, 6000 bism, dans B. PongratzLeisten et al. (éds), Ana šadì Labnani lù allik. Festschrift für Wolfgang Röllig, Alter Orient und Altes testament, Bd 247 (Neukirchen, 1997), p. 47, et surtout Édouard Lipiński, Dieux at déesses de lituivers phénicien et punique, OLA 64, Studia Phoenicia XIV (Leuven, 1995), pp. 319-350.

${ }^{3} 4$ occurrences d'une identification d'Isis à Astarté dans la documentation grecque et latine sont réunies dans Bricault, Myrionymi, p. 15: RICIS 202/0242 i=I. Délos 2101: 130/29 av. J.-C.); RICIS 202/0365 (= I. Délos 2132: $\mathrm{II}^{\mathrm{e}}-\mathrm{I}^{\mathrm{er}}$ siècle av. J.-C.); Étienne Bernand, Inscriptions métriques de l'Ëgpple gréco-romaine (Le Caire, 1969), $\mathrm{n}^{\circ} 175 \mathrm{I}_{1 \mathrm{~B}}$ à Narmouthis dans le Fayoum: ca $84-80$ av. J.-C.; P. Oxy. XI $1380,16-117$ à propos de Sidon: ca 75 apr. J.-C.

Déesse principale de la ville de Sidon (Premier Live des Rois XI, 33; Deuxième Livre des Rois XXIII, 13; Lucien, De dea Syria 4; cf. Mathias Delcor, s.v. Astarté, LIMC III (1986) pp. 1077-1085), Astarté est assimilée à Isis en Égypte depuis au moins le Nouvel Empire; cf. Jean Leclant, «Astartè à cheval d'après les représentations égyptiennesm, Syria XXXVII (1960), pp. 3-4. Plusieurs autres documents plus récents confirment la continuité de ce syncrétisme, comme une statuette d'Isis, de Memphis, sur laquelle est gravée une dédicace en phénicien adressée à Astarté $(R E ́ S$ 535: IV siècle av. J.-C.), ou une stèle, de Memphis encore,-mais la déesse phénicienne y avait son temple, l'Astarticion (cf. Rainer Stadelmann, Syrisch-palästinensische Gottheiten in Aegypten, Probleme der Acgyptologic 5 (Leiden, 1967), pp. 96-110, et notamment pp. 104-105), dédiée à Isis-Astarté (KAI 48: $\mathrm{II}^{e}-\mathrm{I}^{\mathrm{a}}$ siècle av, J.-C,). Cf. également C. Bonnet, Astarte. Dossier documentaire et perspectives historiques, Contributi alla religione fenicio-punica II = Collezione di studi fenici XXXVII (Roma, 1996), pp. 64 et 87.

${ }^{4}$ Isis est assimilée à la déesse Derkéto, l'un des aspects d'Atargatis, dans le 
Déméter. ${ }^{5}$ De même qu'à Cyrène où, dès le IV ${ }^{e}$ siècle av. J.-C., un temple d'Isis s'élevait sur la terrasse de la Myrtousa, dans l'enceinte du sanctuaire d'Apollon, ${ }^{6}$ temple au sein duquel la déesse était vraisemblablement rapprochée de Déméter, et Horus, plus explicitement, d'Apollon, ${ }^{7}$ ainsi à Carthage, l'instauration d'un lieu de culte qui lui soit propre, peut-être dès ce même $\mathrm{IV}^{\mathrm{e}}$ siècle, a pu obéir aux mềmes considérations. ${ }^{8}$ Il n'est alors pas surprenant qu'une inscription, écrite en néo-punique atteste l'existence d'un temple d'Isis et de son prêtre dans cette ville au $\mathrm{III}^{\mathrm{e}}$ siècle av. J.-C., ${ }^{9}$ sinon plus tôt. Épitaphe

P. Oxy. XI $1380_{9596}$, à propos d'Ascalon, dont elle est la principale divinité à l'époque hellénistico-romaine. Elle apparaît fréquemment dans le monnayage de la ville, notamment aux époques antonine et sévérienne, avec des traits qui ne sont pas sans rappeler Isis; cf. Christian Augé, s.t. Derketo, LIMC III, 1 (1986) p. $382 \mathrm{nn}^{\circ} 4-5$.

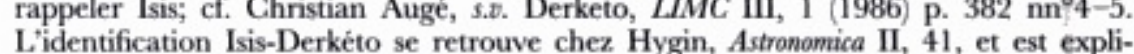
L'identification Isis-Derkéto se retrouve chez Hygin, Astranamica II, 41, et est expli-
quée par le scholiaste de Germanicus (Scholia Basilensia in Germanici Aratea 176,20); quée par le scholiaste de Germanicus (Scholia Basilensia in Germamia Aratea 176,20);
cf. P. L. Van Berg, Corpus Cultus Deae Syriae I. Les sources titteraires, EPRO 28/I (Leiden, 1972), nn ${ }^{\circ} 20-22$ pp. 18-20.

L'identité Isis-Déméter, déjà signalée par Hérodote (II, 59 et 156), est abondamment attestée, notamment en relation avec l'épisode giblite de la quête d'Isis. Cf. Bricault, Mynonymi, pp. 20-21, 79 et 81 et, par exemple, du même, «Isis dolente», BIFAO 92 (1992), pp. 41-42.

- Serena Ensoli Vittozzi, "Indagini sul culto di Iside a Cirenew, dans L'Africa Romana. Atti del IX convegno di Studio Nuoro (Sassari, 1992), pp. 167-250.

RICIS 701/0101 (III' siècle av. J.-C.); Gaspare Oliverio, Documenti antichi dell'Africa italiana I (1927), p. $329 \mathrm{n}^{\circ} 10$ fig. 16; Luisa Vitali, Fonti per la storia della religione gre naica (Padova, 1932), p. 89 n²29 (SEG IX (1944/46) 125); Ensoli Vittozzi, Girene, pp. 187-188 et pl. IV, 1. Il s'agit d'une base de statue, fragmentaire, trouvée a l'ouest du temple d'Apollon, sur la terrasse de la Myrtousa, remployée dans une structure byzantine. Le texte précise qu'un certain Aristis, fils de Théodôros, étant prêtre (d'Apollon), a consacré (la statue) à Horus.

Sur les rapports entre Carthage et la vallée du Nil antérieurs à l'époque grécoromaine, of. Jean Leclant, "Carthage et l'Égypten, dans Actes du IIF Congrès Internationel des Études Phéniciennes et Puniques, Tunis 11-16 novembre 1991 vol. I (Tunis, 1995); pp. 41-50; pour une bonne bibliographie sur la ville de Carthage, voir Pour sauver Carthage, A. Ennabli (éd.) (Paris, 1992).

RICIS 703/0101 (IV ${ }^{e}-\Pi^{e}$ siècles av. J.-C.); Philippe Berger, eInscription funéraire de Melecpalass, dans Musées et collections archéologiques de l'Algérie et de la Tumisie Musé Lavigerie de Saint-Louris de Carthage (Paris, 1900), pp. 35-37, pl. V n 4 (RÉS 13 et 236); M. Lidzbarski, Ephemeris für semitische Epigraphik I (Giessen, 1902), pp. 164-169 CIS 6000 bis et pl. XCVII), James G. Février, L't

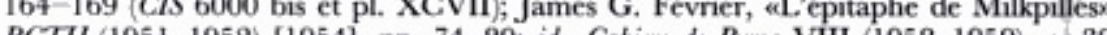
BCTH (1951-1952) [1954], pp. 74-80; id., Cahiers de Byrsa VIII (1958-1959), p. 30; id., *A propos de l'inscription de Milkpillèsw, AION 18 (1968), pp. 195-197; Jean Ferron, eL'épitaphe de Milkpillès à Carthagen, Studi Magrebini l 1 (1966), pp. 67-79 et $\mathrm{pl}$. 1; A. Van den Branden, «Note riguardanti l'iscrizione punica CIS $6000 \mathrm{bis}$, $\mathrm{BeO} 23$ (1981), pp. 155-159; C. Bonnet, art. at. n. 2, pp. 43-56. Il s'agit d'une tablette en calcaire gris dont la partie gauche est détruite; trouvée en mars 1899 dans la nécropole située au nord des citernes de Bordj el Djedid. Elle devait accompagner la stèle funéraire proprement dite, elle aussi épigraphe. Conservée au Musće pagner la stger. d'un prêtre du temple d'Isis apposée sur son monument funéraire par l'un de ses proches, mais non l'un des siens, elle ne nous apprend rien sur la localisation de ce sanctuaire,- le texte provient d'une nécropole et ne nous livre aucune information topographique-, et peu sur son personnel. Le défunt, MLKPLS, est un notable capable de mentionner dans cette inscription funéraire une séquence de 7 ascendants, porteur du titre punique DR ŠPH qu'il semble possible de rapprocher de l'égyptien wr $s 3$ (en grec phylarchos). ${ }^{10}$ Mais il est difficile d'en déduire davantage sur l'organisation religieuse et sociale de ce temple, qui n'a pu en aucune façon précéder le sanctuaire isiaque d'époque impériale, Carthage ayant été détruite en 146 et abandonnée jusqu'au temps de César. Car il n'est toutefois pas encore question de cultes isiaques, ${ }^{11}$ mais de cultes rendus à Isis et, pour Cyrène, à Isis et Horus, divinités proprement égyptiennes. Il n'est pas question d'Harpocrate, d'Anubis et encore moins de Sarapis. Il est d'ailleurs à noter qu'en milieu punique ou phénicopunique, les divinités isiaques sont quasiment absentes. Si l'on considère les territoires sous contrôle carthaginois, le panthéon isiaque semble n'avoir guère rencontré l'adhésion populaire, et encore moins officielle. La carte de répartition des isiaca de Sicile est éloquente: à l'Est hellénisé, où les cultes isiaques sont bien attestés dès le $\mathrm{III}^{\mathrm{c}}$ siècle av. J.-C. s'oppose un Ouest punique vierge de toute présence sérapéenne. En outre, si les influences égyptisantes dans les monnayages de la zone d'influence de Carthage ont depuis longtemps été notées, on constate que la seule Isis - s'il s'agit bien d'elle-, souvent assimilée à Tanit-Déméter, eut le privilège de figurer au droit

${ }^{10}$ C. Bonnet, art. ait. n. 2, pp. 52-53.

"Pour une rapide définition des cultes isiaques, on verra $\mathrm{L}$. Bricault, $\alpha$ Bilan et perspectives dans les études isiaqueso, dans La Grande Dea tra passato e presente, Trop isiaci 1 (Torino, 2000), p. 91. Certes, plusicurs statuettes d'Harpocrate de facture égyptienne portant des inscriptions phéniciennes ont indiscutablement été découvertes en Méditerranée occidentale, et peut-être à Carthage même: cf. J. Ferron, «L inscripción cartaginesa en el Arpocrates madrileñom, Trabajos de Prehistoria 28 (1971) PP. 359-379; id wa statuette d'Harpocrate du Britis Museumow RSF II (1974),

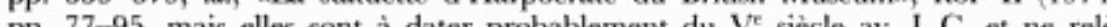
Pp. 77-95, mi vent pas de la diffusion isiaque. Par ailleurs, les «Harpocratesn reconnus par le même auteur sur les stèles puniques de Carthage sont d'identification douteuse. Aux études de J. Ferron, «Horus l'enfant sur les stèles votives de Carthagew, Recue de l'Institut des Belles-Lettres arabes XXXVI, I31 (1973), pp. 79-96; id., "El niño Horus en las estelas votivas de Cartagom, Revista de la Universidad Complutense XXV (janvier-février 1976), pp. 113-126, on pourra objecter que le jeune personnage ne possède aucun des attributs caractéristiques d'Harpocrate, comme la méche de l'enfance, la double couronne (pschent), ou le doigt porté à la bouche. 
de monnaies des $\mathrm{III}^{e}-\mathrm{II}^{e}$ siècles, frappes siculo-puniques pour la Sardaigne, Malte, Cossura. ${ }^{11}$

Il faut attendre le milieu du I ${ }^{\text {er }}$ siècle av. J.-C. pour que la documentation réellement isiaque apparaisse.

Les premiers documents faisant état d'une présence isiaque dans les régions qui nous intéressent ici proviennent de Sabratha, en Tripolitaine. Une monnaie à légende punique au nom de la cité $(\mathrm{SBRT}<\mathrm{N})$ présente en effet au droit Sarapis en buste, barbu, coiffẻ du calathos et au revers la façade d'un temple pentastyle, que l'on pourrait penser être celui abritant le culte public du dieu. La monnaie serait à dater des années $60-50$ av. J.-C., pour des raisons stylistiques et iconographiques. ${ }^{12}$ Sur le plan archéologique, deux temples ont étés reconnus par les archéologues comme isiaques. Le premier serait un temple de Sarapis, ${ }^{13}$ le second un Iséum. ${ }^{14}$ Le premier, situé au Nord-Ouest du forum de Sabratha, est identifié traditionnellement à un Sérapeum sur la foi de la découverte entre ses murs de deux têtes de Sarapis en marbre qui auraient appartenu à des statues de grande taille, la seconde portant une couronne crénelée. Aucune n'a été jusqu'ici publiée et il est bien difficile, non seulement de faire de ce temple un Sérapeum, mais simplement de reconnaître Sarapis sur la seule base de ces brèves descriptions. Qui plus est, le plan établi par Kenrick à partir des travaux de J. B. Ward-Perkins sur ce sanctuaire indique qu'il est hexastyle, ce qui ne correspond pas à l'image livrée par la monnaie au type de Sarapis, pour autant que celle-ci, nous l'avons dit, présente un édifice consacré au dieu. ${ }^{15}$

112 Isis apparait toutefois entre 241 et 238 av. J-C. au droit de monnaies de bronze et de billon émises par les mercenaires libyens insurgés contre Carthage. Cf. Alexandropoulos p. $375 \mathrm{n}^{\circ} 56$ et p. $380 \mathrm{n}^{\circ} 70$.

${ }^{2}$ L. Müller, Numismatique de l'Ancienne Afrique II, pp. 27-29 n ${ }^{\circ} 49$ (Paris, BnF Cabinet des médailles); SVG Copenhagen 36; Jacques Alexandropoulos, Les monnaies de l'Afrique Antique 400 av. J. $-C_{0}-40$ ap. f. $-C$. (Toulouse, 2000), p. 269 et n³9 p. 446.

${ }_{13}$ P. M. Kenrick, Sabratha, JRS Monograph n ${ }^{\circ} 2$ (1986), pp. 115-117 et fig. 53; Véronique Brouquier-Reddé, Temples et culles de Tripolitaine, Études d'Antiquités Africaines 11 (Paris, 1992), pp. 44-48, avec la bibl. antérieure.

it Gennaro Pesce, Il tempio d'Iside in Sabratha, Monografie di archeologia libica 4 (Roma, 1953); Brouquier-Reddé, Temples et cultes, pp. 58-63, avec la bibl. antéricure. Sur la localisation des sanctuaires isiaques de Bulla Regia, Thamugadi, Sabratha et Lepcis Magna, voir Silvia Bullo, "Le indicazioni di Vitruvio sulla localizzazione dei templi urbani (de Arch. I, 7,1): il caso africanom, dans L'Africa Romana X/2 (Sassari, 1992), pp. 526-534, qui résume les travaux antérieurs.

Nous connaissons actuellement deux émissions pour la période pré-impériale, qu'Alexandropoulos daterait de 60-50 av. J.-C. Outre la monnaie au type de Sarapis (c. supra $\mathrm{n} .12$ ), l'autre présente au droit une tête barbue et laurée (Zeus?), tandis
L'identification traditionnelle n'est donc guère convaincante. ${ }^{16}$ La présence d'un culte civique de Sarapis ne fait pourtant aucun doute au tournant de l'ère chrétienne. Plusieurs émissions monétaires sont à l'effigie du dieu, qui apparaît ainsi au revers de monnaies d'Auguste et de Tibère. ${ }^{13}$

Le second temple pose lui aussi problème. Situé dans l'insula 15 de la regio III, en bord de mer, il fut fouillé par les archéologues italiens entre 1934 et 1947. La publication de ces fouilles montre qu'à un petit temple érigé sur un podium - aujourd'hui à l'ouest du second temple, sous le sol de la cour - a succédé un sanctuaire plus vaste. Toutefois, aucun document clairement isiaque n'est antérieur à la fin du $\mathrm{II}^{\mathrm{e}}$ siècle apr. J.-C., ce qui peut laisser penser que le premier édifice n'était pas nécessairement voué à Isis ou à Sarapis. Il est d'ailleurs bien difficile d'attribuer la primauté dans le second temple à l'une ou l'autre des divinités, malgré le titre de la monographie de Pesce. On a voulu en restituer la dédicace (à Isis) à partir de trois fragments publiés par Pesce, complétés par de nouvelles trouvailles, et datés du règne de Vespasien. Or il n'en est rien. ${ }^{18} \mathrm{En}$

qu'au revers figure un temple, hexastyle celui-là: Alexandropoulos, Monnaies, $n^{\circ} 38$ p. 445 .

${ }_{16}$ Elle est wacceptée par tous les auteurss selon Brouquier-Reddé. Remarquons toutefois que J. B. Ward-Perkins, comme le rapporte Kenrick (op. cit. n. 12, p. 115 n. 31) émettait des réserves, indiquant que eThe identification of this temple seems very far from certainm; de son côté, Wild, Isis-Sarapis Sanctuaries, pp. 1817-1818, s'il ne récuse pas cette identification, note le peu d'éléments dont nous disposons pour assurer celle-ci.

RPC I n 811 = Alexandropoulos, Monnaies, $\mathrm{n}^{\circ} 40$ p. 446 (D/tête nue d'Auguste, légende punique au nom de la ville SBRT[...]; R/tête de Sarapis: vers 8-5 av. J.-C. ?); $n^{\circ} 822=$ Alexandropoulos, Mompies, $n^{\circ} 41$ P. 446 (D/Sarapis debout de face, J-C... n 822 - Alcxandropoulos, Monnaies, n 41 p. 4 (D/Sarapis debout de face, droite levee et tenant un sceptre de la gauche, legende punique au nom de la ville SBRTN; R/Capricorne, cornucopia, gouvernail et globe: vers 8-5 av. J.-C.?); $\mathrm{nn}^{\circ} 814-815=$ Alexandropoulos, Monnaies, $\mathrm{nn}^{\circ} 43 \mathrm{a}-\mathrm{b}$ p. 447 (D/tête nue d'Auguste, légende CAESAR; R/tête de Sarapis, légende punique au nom de la ville (SBRT $<\mathrm{N}$ ) avec nom de magistrat, lui aussi en punique ( $R$ pour la 814 , S Y MN pour la 815 : 7-6 av. J.-C. pour les auteurs du RPC ou 8-14 apr. J.-C. (?) pour Alexandropoulos); nn 817 et $820=$ Alexandropoulos, Monnaies, $\mathrm{nn}^{\circ} 43 \mathrm{~d}$-c p. 447 (D/tête radiée d'Auguste; $R$ /tête de Sarapis, légende punique au nom de la ville (SBRT<N) avec

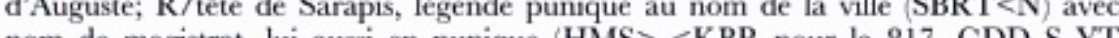
MN pour la 820: règne de Tibère pour les auteurs du $R P C$ ou 8-14 apr. J.-C.(??) pour Alexandropoulos).

${ }^{18}$ Déjà mentionnée par E. Vergara Caffarelli, FA 12 (1957 [1959]), p. 354 n 5621 (AE, 1959, 69b; 1961, 143), cette tentative de restitution est exploitée par G. di Vita-Evrard, «La dédicace du temple d'Isis à Sabratha: une nouvelle inscription africaine à l'actif de C. Paccius Africanusw, LibAnt 3-4 (1966-1967), pp. 13-20 et pl. 2-4, et admise par Brouquier-Reddé, pp. 62-63; pour ingénieuse qu'elle soit, cette restitution ne convainc pas; elle est combattue déjà par Herbert W. Benario, cette restitution ne convainc pas; elle est combattue deja par Herbert W. Benario,
aCaius Paccius Africanus at Sabrathas, Epigraphica 28 (1966), pp. 135-139, qui a 
l'état actuel de nos informations, le temple a abrité une statue de grande taille d'Isis debout tenant la situle ${ }^{19}$ et une autre de Sarapis trônant, qui ont pu être les statues de culte, et que l'on peut dater de la fin du $\mathrm{II}^{\mathrm{e}}$ ou du début du $\mathrm{III}^{\mathrm{e}}$ siècle apr. J.-C. ${ }^{20}$ Une statuette en marbre d'Isis, une petite tête d'Harpocrate (sans doute du $\mathrm{I}^{\mathrm{er}}$ siècle apr. J.-C. pour cette dernière), deux lampes bilychnes à buste de Sarapis ${ }^{21}$ y ont également été trouvées. Deux autres du même type, bilychnes, ont été découvertes dans des maisons. ${ }^{22}$ Le dieu en buste à droite est présent sur deux autres lampes du $\mathrm{III}^{\mathrm{e}}$ siècle, ${ }^{23}$ alors qu'il est lampadophore sur six autres exemplaires. ${ }^{24}$ À noter qu'il n'apparait pas sous sa forme solaire. Isis est présente sur une lampe tardive, ${ }^{25}$ ainsi que sur une lampe naviforme dont il ne subsiste que la proue. ${ }^{26}$ Enfin, une dédicace très fragmentaire à Isis gravée sur une plaque de marbre blanc a été découverte dans la voûte sud de la cella, ${ }^{27}$ mais rien n'implique qu'il s'agisse d'une inscription officielle livrant le nom de la divinité principale. Il peut tout aussi vraisemblablement s'agir d'un acte de dévotion privé. Faire de ce temple un sanctuaire isiaque est juste, en faire un Iséum revient à extrapoler la documentation.

Sur les rivages de la grande Syrte, en remontant vers le nord, Thaenae se présente également, aux premiers temps de l'ère chré-

montré qu'il s'agirait plutôt de la dédicace à Vespasien d'un arc de triomphe, et rejetée par Vidman, SIRIS 795 et Wild, Isis-Sarapis Sanctuaries, p. 1817.

${ }^{19}$ Pesce, Sabratha, pp. 49-50, n०21 fig. 28; Eingartner, Isis und lhre Dienerinnen, p. $133 \mathrm{n}^{\circ} 67 \mathrm{et} \mathrm{pl}$. XLV, que l'on date de 210 apr. J.-C. environ.

${ }_{20}$ Voir les remarques pleines de bon sens de Wild, Isis-Sarapis Sanctuaries, p. 1818 , sur la disposition des statues et l'emplacement des chapelles ayant dû les abriter.

E. Joly, Luceme del Museo di Sabratha, Monografie di archeologia Libica 11 (Roma, 1974), pp. 76-77 et $107 \mathrm{nn}^{\circ} 79-80$ et pl. VIII

${ }_{22}$ Ibid., $\mathrm{nn}^{\circ} 78$ et 81 , p. 107 et pl. VII.

${ }^{23}$ Ibid., nn ${ }^{\circ} 678-679$, p. 151 et pl. XXV.

${ }^{24}$ Ibid., nn ${ }^{\circ} 850-855$, pp. 165-166 et pl. XXXIV,

25 Ibid., n866, p. 167 et pl. XXXV, à comparer avec celles de Sbeitla et d'Haidra

qui sont entières alors que celle-ci est fragmentaire.
${ }_{26}$ Ibid., n839 p. 164 et pl. XXXI. D'autres lampes signalées par Joly sont peu convaincantes: l'une d'Isis en buste de face $\left(\mathrm{n}^{\circ} 637 \mathrm{p}\right.$. $148 \mathrm{et}$ pl. XXIV), une autre d'Isis debout de face avec corne d'abondance et patère ( $\mathrm{n}^{\circ} 569 \mathrm{p} .143$ et pl. XXIII), cinq d'Isis debout à côté d'un autel (nn507-511 p. $138 \mathrm{et} \mathrm{pl}$. XX), une d'Isis dans un temple $\left(\mathrm{n}^{\circ} 722\right.$ p. $155 \mathrm{et}$ pl. XXVII).

${ }^{27}$ RICIS 702/0201 (III' siècle apr. J-C.?); Reynolds et Ward Perkins, Inscriptions of Roman Tripolitania $\mathrm{n}^{\circ} 8$; SIRIS 796. Musée de Sabratha. Sur certaines des fonctions supposées de ce temple, on verra J. Eingartner, «Bemerkungen zur Funktion römischer Tempel am Beispiel des Isisheiligtums in Sabratha und des sogennanten Serapeion in Ephesoss, dans L'Africa Romana 13, Djerba 1998, vol. 2 (Roma, 2000), pp. 1211-1221. tienne, comme un centre de culte isiaque. Plusieurs monnaies de l'époque augustéenne montrent en effet au droit la tête de Sarapis, ${ }^{28}$ comme à Sabratha. Le nom du proconsul apparaît au revers, ce qui doit indiquer qu'il s'agit d'un culte public. De ce site proviennent une lampe d'Isis et Héliosarapis affrontés ${ }^{29}$ et, de la nécropole, une autre à buste de Sarapis à gauche. ${ }^{30}$

Mentionnons également une monnaie au type de Sarapis, datable vraisemblablement, pour des raisons métrologiques, du milieu du $I^{\text {er }}$ siècle av. J.-C., mais d'attribution incertaine. Si certains arguments rappelés par J. Alexandropoulos suggèrent d'en rapporter la frappe à l'atelier de Sabratha, plusieurs indices invitent à l'attribuer, sans certitude, à la cité de Zita. ${ }^{31}$

Enfin, deux dernières monnaies posent réellement problème. Elles sont attribuées, non sans hésitations, à Hadrumète par J. Alexandropoulos parce que leur droit présente «'effigie d'un dieu à tiare, sans doute Baal Hammon", dont le culte hadrumétain est bien connu, effigie qu'il rapproche de celle du dieu que l'on retrouve cette fois indiscutablement sur une monnaie d'époque augustéenne $(6-5$ av. J.-C.), d'attribution certaine à Hadrumète. ${ }^{32}$ Cette double émission, que leur métrologie semble rapprocher du quart et du demi-shekel, est datée du $\mathrm{II}^{e}$ siècle av. J.-C. par G. K. Jenkins, datation acceptée par J. Alexandropoulos. ${ }^{33}$ Le rapprochement avec la monnaie

28 RPC I n ${ }^{\circ} 807=$ Alexandropoulos, Monnaies, $\mathrm{n}^{\circ} 53$ p. 450 (D/tête de Sarapis, légende punique au nom de la ville $\mathrm{T}<\mathrm{YNT}$; $\mathrm{R} /$ tête d'Astarté(?') diadémée, même légende T<YNT: vers 3 apr. J.-C. pour Alexandropoulos p. 278) et $810=$ Alexandropoulos, Monnaies, n 55 p. 450 (D/buste de Sarapis, légende punique au nom de la ville $\mathrm{T}<\mathrm{YNT}$; $\mathrm{R} /$ tête nue, barbue du proconsul $\mathrm{A}$. Vibius Habitus, ave légende latine P A VIBI HABITI PRO CO[S]: 16-17 apr. J.-C. pour B. E. Thomasson, Die Statthalter Nordafrikas (Lund, 1960) et Stumpf, aEine Porträtmünze des A. Vibius C. f. C. N. Habitus, Proconsul von Africa unter Tiberiuss, Schweize Münzblatter 33, n¹30 (Mai 1983), pp. 33-35, 13-14 apr. J-C. pour les auteurs du $R P C$ et Alexandropoulos). Gerd Stumpf ne se prononcait curieusement pas sur le nom de la cité émettrice, alors que la légende de la monnaie qu'il publiait était relativement lisible.

${ }^{29}$ H. de Villefosse, $B S A F(1881)$, p. 234.

so R. Massigli, Musées et collections archéologiques de l'Algérie et de la Tunisie. Musée de Sfax, n ${ }^{\circ} 91$ p. 35.

${ }^{31}$ Cr. SNG Copenhagen n ${ }^{\circ} 47$ et déjả, L. Müller, Numismatique de l'Ancienne Afrique II, p. 59 et III n ${ }^{\circ} 68$, qui suggérait Suthul plutôt que Zita; voir les remarques prudentes et judicieuses d'Alexandropoulos, Monnaies, p. 273 et $n^{\circ} 47$ p. 448 (D/tête de Sarapis; R/légende punique ST dans une couronne de lauriers).

Sarapis; R/légende punique ST dans une
${ }_{12}$ Alexandropoulos, Monnaies, p. 288.

${ }^{32}$ Alexandropoulos, Monnaies, p. 288.
ss G. K. Jenkins, dans SNG Copenhagen n ${ }^{\circ} 68$; L. Müller, Numismatique de lAncienne Afrique III, p. $71 \mathrm{n}^{\circ} 83$, qui ne connaissait que le quart de Shekel, classait cette monnaie en Numidie; J. Mazard, CNNM, p. $206 \mathrm{n}^{\circ} \mathrm{XXXV}$ la classait parmi le 
d'époque impériale, pour ingénieux qu'il soit, n'est pas entièrement convaincant, le couvre-chef ressemblant davantage à un calathos qu'à une tiare. Il est alors tentant de retrouver sous ces traits divins Sarapis plutôt que Baal Hammon, à moins que l'on n'ait affaire à un subtil syncrétisme entre les deux iconographies. L'identification du dieu à Sarapis ne remettrait d'ailleurs pas en cause l'attribution de cette frappe à Hadrumète, la présence d'un culte public du parèdre d'Isis, bien attestée dans les autres emporia de la côte, ne pouvant surprendre dans ce port actif et cosmopolite.

Il faut attendre le début du $\mathrm{II}^{\mathrm{e}}$ siècle apr. J.-C. pour voir la documentation s'accroître considérablement. Trois nouveaux sanctuaires au moins, d'époque antonine, sont attestés pour la Tripolitaine et la Proconsulaire.

À Lepcis Magna, un temple de Sarapis fut découvert en 1960, entre le marché et le vieux forum, à l'angle d'une insula. ${ }^{34}$ Fouillé à plusieurs reprises par les archéologues italiens, il a livré un matériel très abondant, presque totalement inédit. Sa date de construction reste difficile à déterminer en l'absence de renseignements précis. Toutefois, la statue de culte de Sarapis, qui présenterait les traits d'Antonin le Pieux, et une statue de Marc Aurèle permettraient de situer l'édification du sanctuaire au moins à l'époque antonine, plutôt que sévérienne, comme on le pensait sur la foi des seules six inscriptions publiées jusqu'alors. ${ }^{35}$ Plusieurs autres statues (de Sarapis, d'Isis, etc.), une trentaine d'inscriptions en caractères grecs, dont deux, gravées sur les côtés de l'escalier menant au temple, rapportent la dédicace de deux statues de Sarapis, tandis que d'autres encore s'adressent à Sarapis et à ses sunnaoi theoi, font du dieu la principale divinité du sanctuaire. L'immense majorité des inscriptions, la céramique retrouvée lors des fouilles, plusieurs statues ${ }^{36}$ indiquent que l'apogée des

incertaines. Alexandropoulos, Monnaies, n ${ }^{\circ} 78$ p. 455 la décrit ainsi: D/buste drapé de Baal Hammon, coiffé d'une tiare; légende punique $>\mathrm{T}$; R/lion marchant à droite, légende punique BT. Nous avons pu recenser 3 autres exemplaires de ce type, passés récemment dans le commerce: Catalogue J. Malter du 28 mars 1999 p. $10 \mathrm{n}^{\circ 258}$; Catalogue Lanz 102 du 28 mai 2001, n³48; Catalogue Classica Numismatic Group 58 du 19 sept. 2001, nº741.

s4 Cf. Brouquier-Reddé, Temples at cultes, pp. 101-105.

s5 SIRIS $797-802=$ RICIS $702 / 0101-0106$, toutes datées du III ${ }^{e}$ siècle.

Cf. Eingartner, Isis und ihre Dienerinen, p. $114 \mathrm{n}^{\circ} 12 \mathrm{et} \mathrm{pl}$. XI, ainsi que p. 127 $\mathrm{n}^{\circ} 48 \mathrm{ef}$ pl. XXXII pour deux grandes statues d'Isis retrouvées dans les thermes de Lepcis. La première daterait des années 120-130, la seconde de la décennie suivante. cultes isiaques à Lepcis Magna est à situer dans le premier tiers du $\mathrm{III}^{\mathrm{e}}$ siècle, lorsque ceux-ci ont bénéficié de la faveur des Sévères et notamment des initiatives que prit le fondateur de la dynastie pour embellir sa ville natale. Sur l'arc de Septime Sévère, l'Empereur s'est fait représenter en Sarapis trônant, selon le type iconographique de la statue cultuelle d'Alexandrie, tandis que son épouse Julia Domna est figurée en Junon. La plupart des fidèles de cette époque - les renseignements font défaut pour le $\mathrm{I}^{\mathrm{er}}$ siècle et le début du $\mathrm{II}^{\mathrm{e}}$ siècle-, sont des Grecs originaires d'Orient, voire d'Égypte, et non des Latins.

Non loin de là, à Sahel di Homs, fut découverte une stèle de marbre en forme de naos distyle, dont le fronton triangulaire décoré aux extrémités d'acrotères grossiers, s'orne en son centre d'un buste de Sarapis coiffé du calathos. Au-dessous, entre les deux colonnes ioniques, un serpent dressé à droite, gueule ouverte; devant lui, un pilier cylindrique supporte une pomme de pin; la base de la stèle représente le podium d'accès au temple. ${ }^{37}$ Sur les rampants du fronton, le bandeau, les colonnes et la base du naos court une dédicace à Asclépios pour la victoire de souverains qui doivent être Septime Sévère et ses fils. Le dédicant, un marbrier du nom d'Asclépiadès, fils d'Asclépiadès, identifie clairement Sarapis et Asclépios. L'attachement familial du marbrier au dieu Asclépios rejoint ici d'une manière remarquable celui de la famille sévérienne pour Sarapis et trouve son aboutissement dans l'identification révélée par cette stèle.

Autre fondation d'époque antonine, le temple isiaque de Bulla Regia. Dégagé lors de fouilles menées par l'INP en 1959-1962, il se trouve au Sud du théâtre. ${ }^{38}$ On y accède par une placette faisant partie d'une vaste esplanade fermée, où l'on dénombre plusieurs sanctuaires. Un autel dédié à Isis, ${ }^{39}$ une statue de la déesse qui pouvait être la statue de culte permettraient d'en faire un Iséum. Les deux documents seraient datables du milieu du $\mathrm{II}^{c}$ siècle. Ces mêmes fouilles ont livré une petite tête d'enfant porteur de la mèche d'Horus,

${ }^{37}$ RICIS 702/0401. Musće de Lepcis Magna. III siècle apr. J.C. [198-211 apr. J.-C.?].

Azedine Beschaouch, Roger Hanoune, Yvon Thébert, Les nuines de Bulla Regia, Collection de l'École Française de Rome 28 (1977), p. 107 et fig. 106 pour une vue du sanctuaire.

RICIS $703 / 0401$ 
œuvre du début du $\mathrm{III}^{e}$ siècle. ${ }^{40}$ De la même époque datent de nombreuses lampes retrouvées en divers endroits de la ville. ${ }^{41}$

Il est temps de revenir à Carthage. Les trouvailles éparses de Sainte-Marie, à la fin de 1874, «entre la colline de Saint-Louis [i.e. la colline de Byrsa] et les grandes citernes de Bordj el Djedid ${ }^{42}$ invitaient à y localiser un sanctuaire isiaque d'époque impériale.$^{+3}$ Depuis, d'autres découvertes très probablement in situ - une tête colossale de Sarapis et une inscription bilingue dédiée à Isis et Sérapis $^{\text {H4 }}$ permettent de le localiser plus précisément entre les thermes d'Antonin et le théâtre, dans l'insula délimitée par le decumanus II nord et les kardines XI et XII est, de part et d'autre de la ligne du T.G.M. Une dizaine d'inscriptions, ${ }^{45}$ de multiples fragments statuaires, des lampes à type isiaque ${ }^{46}$ invitent à considérer que Sarapis en fut le principal occupant. ${ }^{47}$ Les nombreuses statues, souvent de belle facture, retrouvées dans l'aire du sanctuaire mais aussi ailleurs dans la cité sont la marque d'un culte florissant aux $\mathrm{II}^{\mathrm{e}-} \mathrm{III}^{\mathrm{e}}$ siècles apr. J.-C. ${ }^{48}$ Le

to Cf. Nayla Attya Ouertani, «Deux documents relatifs au culte d'Isis à Bulla Region, dans L'Afrique du Nord antique et médićale, $\mathrm{VI}^{\leftarrow}$ colloque international sur l'histoire et l'archéologie de l'Afrique du Nord, Pau octobre 1993 (1995), pp. 395-404. Les deux fragments statuaires ont été retrouvés dans une cache à l'intérieur du péribole du temple.

41. Cf. infra nn. 62-64.

12 Stéphane Gsell, "Les cultes égyptiens dans le Nord-Ouest de l'Afrique sous l'empire romainn, RHR 30 tome 59 (1909), p. 149; voir à ce sujet Auguste Audollent, Carthage romaine (Paris, 1901), pp. 238-240 et 402-408.

${ }^{4}$ Sur ce temple, voir les données anciennes réunies par Wild, Isis-Sarapis Sanctuaries,
Sume (Paris, 1901 ), pp. $238-240$ et $402-408$. p. 1763. Jean-Pierre Laporte prépare la publication très attendue de l'abondant matériel retrouvé par Évariste Pricot de Sainte-Marie, dont la majeure partie se trouve soit au Musée d'Alger, soit au Musée du Louvre. En attendant, on peut toujours se reporter au bref inventaire établi par Gsell, art. cit. n. 42, pp. 149-152.

${ }^{*}$ RICIS 703/0112. Cet important document a été publié par A. Beschaouch,

"Sur la localisation du temple d'Isis. Topographie de Carthage romaines, CRAI

1991,2 , pp. 323-330 et figg. 1-3 (AE, 1991, 1662a-b).

is Les inscriptions RICIS 703/0101-10 proviennent toutes de ce site.

*6. Cf. infra pp. 233-234.

47 Il est done difficile de parler d'Iséum de Carthage, à la suite d'A. Beschaouch.

48 Cf. Bricault, Atlas, p. 84. À noter en outre une statuette en bronze d'Isis drapée de $35 \mathrm{~cm}$ (Musée du Bardo $\mathrm{n}^{\circ}$ inv. F 367; Merlin, Musée Alaota suppl. 2, p. 140; M. Yacoub, Le Musée du Bardo (Tunis, 1969), p. 58) ainsi qu'une statuette en bronze de Jupiter Sarapis assis, vêtu d'un manteau, le bras droit baissé et le gauche levé de $7 \mathrm{~cm}$ (Musée du Bardo ${ }^{\circ}$ inv. F 368; Merlin, Musée Alaoui suppl. 2 p. 140; Yacoub, Musé du Bardo, p. 72). Ces deux bronzes ont été trouvés en 1910 dans les citernes de Dar-Saniat, à Carthage. Signalons en outre une intaille en cornaline d'Harpocrate assis sur un autel, l'index droit à la bouche, tenant le sceptre heka sur l'épaule gauche, en provenance de Carthage (L. Hautecoeur, Musée Alaoui, supplément, N 80, p. 343; Yacoub, Musée du Bardo, pp. 57-58). À ceci, il faut peut- panthéon isiaque y est alors assez large puisque outre Isis et Sarapis, sont vénérés Anubis, Harpocrate, Osiris et même Hermanubis. Cependant, ici comme dans l'ensemble des provinces africaines, Apis n'apparaît pas. Plusieurs dédicaces émanent de représentants d'une même famille, les Aurelii Pasinici. L'une de leurs offrandes est remarquable. Il s'agit d'une proue de navire soutenant un pied droit levé, vestige probable d'une grande statue de Sarapis-Neptune comme l'indique la dédicace inscrite sur celle-ci. ${ }^{99} \mathrm{Ce}$ document doit pouvoir être mis en relation avec le rôle de protecteur de la navigation assumé par Sarapis à l'époque impériale. Associé à Isis, il est notamment chargé du bon acheminement de l'annone à Rome, dont on sait qu'elle est pour une bonne part, et de plus en plus au fil du temps, de provenance africaine. ${ }^{50}$ Autre aspect du dieu à l'honneur dans le sanctuaire carthaginois, celui de guérisseur. Une dédicace, fragmentaire, est en effet adressée par les deux mêmes dédicants que la précédente à [Sarapis?], dieu très grand de Canope. ${ }^{51}$ Ce dernier porte également à deux reprises son «épiclèse canonique» des époques antonine et sévérienne de Zeus Hélios grand Sarapis. ${ }^{52}$ À Carthage, il est bien un dieu panthée. ${ }^{53}$ La présence dans le sanctuaire d'effigies impériales, dont une belle et fameuse statue de Sabine,

être ajouter une statue acéphale d'Isis conservée au Rijksmuseum de Leiden ( $\mathrm{n}^{\circ}$ inv. 1826, H III T2 (TU)), publice en dernier licu par Eingartner, Isis und ihre Dienerinnen, p. $118 \mathrm{n}^{\circ} 23$ et pl. XIX, que cet auteur date du règne de Marc Auréle.

49 CIL VIII, $1002=12462=$ SIRIS $770=$ RICIS 703/0102. Pour un recensement prosopographique des isiaques d'Afrique du Nord (Cyrénaĭque comprise), on pourra se référer à Mora, Prosopografia Isiaca, pp. 509-521, dont le dépouillement s'est interrompu en 1987. On regrettera seulement que l'auteur de cet ouvrage si utile ait compté au nombre des fidèles les empereurs mentionnés dans les inscriptions.

to Sur l'annone en général, on verra les études récentes de G. E. Rickmann, The com supply of ancient Rome (Oxford, 1980), A. J. B. Sirks, Food for Rome. The Legal Structure of the Transportation and Processing of Supplies for the Imperial Distributions in Rome and Constantinople (Amsterdam, 199l), ainsi que les contributions au volume Le ratitaillement en blè de Rome, E. Gabba (éd.) (Napoli-Roma, 1994), qui fournissent une abondante documentation sur le sujet.

Sur ce rôle de protecteur de la navigation, ef. en dernier lieu L. Bricault, «Un phare, une flotte, Isis, Faustine et l'annonen, CdÉ LXXV (2000), pp. 136-149.

${ }^{1}$ CIL VIII, $1003=$ SIRIS $771=$ RICIS 703/0103. La dédicace offre la particularité d'être rédigée en grec, alors que la dédicace à Sarapis-Neptune est en latin. Mais nous avons déjà vu que le bilinguisme semble de mise dans ce sanctuaire. Sur Sarapis de Canope, cf. l'étude à paraitre de L. Bricault, «Sarapis de Canope et Isis de Ménouthis, les véritables Osiris-Canopem.

${ }_{32}$ CIL VIII, $1005=$ SIRIS $774=$ RICIS $703 / 0106$ et CIL VIII, $12493=$ SIRIS $777=$ RICIS 703/0110. Sur le sens et l'origine de cet aspect divin, cf. L. Bricault, ¿Zeus Hélios mégas Sarapis» (à paraître).

is RICIS 703/0110. 
épouse d'Hadrien, figurée en Cérès ${ }^{54}$ apporte de nouveaux éléments à intégrer dans le riche dossier des rapports existant entre le pouvoir impérial, les divinités isiaques et l'annone, que nous avons déjà évoqués ailleurs ${ }^{55}$ et sur lesquels nous reviendrons prochainement plus longuement. Sur le personnel du sanctuaire, nous sommes peu renseignés. Le seul membre du clergé qui nous soit connu est un certain Tiberius Claudius Sarapiacus, prêtre, probablement un pérégrin. ${ }^{56}$

Aucun autre sanctuaire n'est attesté pour le moment en Proconsulaire. Plusieurs documents, toutefois, permettent de supposer une diffusion plus dense des cultes isiaques. Ici comme ailleurs, les premières traces d'implantation isiaque se repèrent dans les ports: Lepcis Magna, Sabratha, Thaenae et Carthage. Le rôle des marchands dans cette diffusion a souvent été souligné. Il est essentiel, mais on ne doit pas négliger non plus celui des migrants - quel que soit leur rang social-, naissant ici pour mourir là, et transportant avec eux coutumes et croyances. Dans ces régions, le double emploi du grec et du latin dans les dédicaces doit renvoyer à ce même brassage de populations, dans ces cités côtières cosmopolites où des individus venus de partout s'installent un temps, se croisent, puis repartent. D'autres cités du rivage des Syrtes ont livré des témoignages isiaques, insuffisants en l'état pour en faire d'importants foyers de dévotion, mais qu'il est bon de signaler. À Gigthis, on a retrouvé une tête colossale de Sarapis en marbre,,$^{57}$ une de la déesse Isis ${ }^{58}$ ainsi qu'une lampe naviforme avec Isis au centre et Sarapis à la poupe,,$^{59}$ copie conforme d'une lampe retrouvée à Carthage. ${ }^{60}$ Ces documents, aux-

अ Cf. Kate De Kersauson, «La Sabine "Pricot de Sainte-Marie" ", RLouvre XLVII/2 (1997), pp. 27-35 au sujet de cette belle statue, dont la tête a été retrouvée récemment au fond de la rade de Toulon, à l'initiative de Jean-Pierre Laporte.

ss Cf. Bricault, art. cit. n. 50.

s6 CIL VIII, $1004=$ SIRIS $773=$ RICIS 703/0105. 37 Gsell, art. cit. n. 42, p. 152; of. Mariangela Pisanu, «La vita religiosa a Gigthis:
testimonianze epigrafiche e monumentalin, dans LAfrica romana VII (Sassari, 1989), pl. III. Sur les aegyptiaca découverts à Gigthis, voir P. Gauckler, «Les fouilles de Tunisien, RA (1902/2), p. 401; L. Poinssot, Catalogue des musées el collections archéologiques de l'Algérie et de la Tunisie: Musée Alaoui. Supplément (Paris, 1910), p. 59, C 1031; Yacoub, Musée du Bardo, p. 84; Wild, Isis-Sarapis Sanctuaries, p. 1779.

${ }_{58}$ Yacoub, Musée du Bardo, p. 48, C 1032, d'après Poinssot, Musée Alaoui. Supplément (1910), p. 59. À noter que Gauckler, CRAI (1903), pp. 462-463, évoque un temple de Sarapis, un autre d'Isis dans le quartier de la marine, des têtes colossales de Sarapis, des têtes d'Isis à Gigthis sans étayer ses affirmations.

${ }^{59}$ Musée du Bardo ${ }^{\circ}$ inv. K 1281; signalée dans L'Africa ramana VII (Sassari, 1989), p. 230.

${ }_{60}$ Lampe naviforme à huit becs; Sarapis en buste est à la poupe, Harpocrate en quels il convient d'ajouter la lampe fragmentaire de Sabratha, ${ }^{61}$ viennent renforcer l'un des aspects primordiaux de la famille isiaque en Afrique, celui de protecteurs de la navigation que nous avons évoqué plus haut. Il est probable que la célébration du Navigium Isidis, le 5 mars, marquait ici aussi l'ouverture de la navigation. N'oublions pas que nous devons à Apulée la description la plus vivante et la plus détaillée de ces cérémonies, et qu'il est tentant de supposer que c'est dans l'une de ces cités portuaires que l'écrivain de Madaure vit pour la première fois le cortège isiaque qu'il décrira plus tard au livre XI des Métamorphoses.

Le nombre de lampes retrouvées sur le territoire de la Proconsulaire est important. Il n'est malheureusement pas toujours possible de déterminer leur type exact car elles ont fréquemment été signalées dans d'anciennes publications sans que la forme de la lampe ait été indiquée.

À Bulla Regia, on peut dater les lampes d'Isis et Anubis, ${ }^{62}$ de Sarapis et Isis conjugués ${ }^{63}$ et d'Héliosarapis et Isis affrontés ${ }^{6 t}$ de la période allant du milieu du $\mathrm{II}^{\mathrm{e}}$ au milieu du $\mathrm{III}^{\mathrm{e}}$ siècle. À Hippo Regius, des fragments de deux lampes des dieux affrontés ${ }^{65}$ offrent la même datation. À Calama, on retrouve le couple divin ${ }^{66}$ mais aussi Sarapis en buste tourné vers la gauche. ${ }^{67}$

Le site d'Hippo Diarrhytus a livré une nouvelle lampe du couple. ${ }^{68}$ À Carthage, les lampes sont plus nombreuses, en provenance d'un magasin ou d'un dépôt tout proche du sanctuaire isiaque près du

buste à la proue; au centre, l'emplacement réservé à Isis est vierge (Musée du Bardo $\mathrm{n}^{\circ}$ inv. $\mathrm{K} 1280$ ).

6) Cf. supra n. 26 .

${ }_{62}$ Musée du Bardo $\mathrm{n}^{\circ}$ inv. K 134: J.-L. Podvin, «Anubis et Isis sur des lampes à huile romaineso, RLouve $(2001 / 4)$, pp. 33-36.

${ }^{65}$ L. Carton, «La nécropole de Bulla Regian, BCTH 1890, pp. 212-213. La lampe, non identifiée comme telle par Carton, porte trois cyprès sur le fond.

${ }^{6+}$ Musée du Bardo $\mathrm{n}^{\circ}$ inv. K 135: Du Coudray La Blanchère, P. Gauckler, Musées el collections archéologiques de l'Algére et de la Tunisie. Musée Alavui (1897), p. 162. Elle porte la marque SER au revers.

6s J. Bussière, Lampes antiques d'Algérie, pp. 169 et 409, n $7369 \mathrm{pl} .154$ pour l'une, A. Delamare, Exploration scientifique de l'Algérie pendant les annies 1840-1845, pl. 193 n 3-4 pour l'autre.

${ }^{6}$ Bussière, op. cit., pp. 169 et $361, n^{\circ} 3517$ et pl. 97.

67 A. Delamare, op. cat., pl. $182 \mathrm{n}^{\circ} 22$. Ce type de lampes se trouve du milieu du $\mathrm{I}^{\mathrm{I}}$ au milieu du $\mathrm{III}^{e}$ siècle.

6. L. Budde, “Julian-Helios Sarapis und Helena-Isis», $A A 87$ (1972), fig. $30 \mathrm{pp}$. 640-641, repris dans Iside, IV.309, p. 274. 
Bordj el Djedid: une anse en forme de buste du dieu, ${ }^{69}$ vingt-deux lampes identiques d'Isis et Sarapis conjugués, ${ }^{70}$ deux d'Isis et Anubis. ${ }^{71}$ Des nécropoles, on peut ajouter une lampe d'Harpocrate, ${ }^{72}$ une à buste de Sarapis à gauche ${ }^{73}$ à dater entre le milieu du $\mathrm{I}^{\mathrm{er}}$ siècle et la fin du II $^{e}$ siècle et la lampe naviforme à huit becs figurant Harpocrate et Sarapis en buste. ${ }^{74}$ D'endroits non précisés à Carthage proviennent un fragment du même Sarapis en buste, ${ }^{75}$ une lampe d'Héliosarapis de face, ${ }^{76}$ trois du couple divin affronté, ${ }^{77}$ une d'Isis et Anubis, ${ }^{78}$ deux d'Harpocrate ${ }^{79}$ et une de la triade Harpocrate, Isis, Anubis. ${ }^{80}$

Une lampe à buste isiaque a été relevée dans le sanctuaire de Saturne au Djebel bou Kournein. ${ }^{81}$ Pour Tunis, on évoquera une lampe de Sarapis trônant. ${ }^{\text {.2 }}$

Hadrumète constitue un centre privilégié pour les lampes isiaques.

69 J. Deneauve, Lampes de Carthage (Paris, 1969), p. 219, n¹101 et pl. C.

70 lbid., pp. 202-203, nn ${ }^{\circ} 981-982$ et pl. LXXXIX pour deux de ces lampes. A.-L. Delattre, "Lampes romaines trouvées à Bordj-Djedid", Revue tunisienne (mars 1913), p. $189, \mathrm{n}^{\circ} 10$, indique que ale même sujet se retrouve sur une vingtaine de lampess, dont la n981 citée, portant la marque CHEL. La n ${ }^{\circ} 982$ est fragmentaire,
et a été trouvée à Carthage, sans autre précision. On peut encore ajouter la lampe et a été trouvée à Carthage, sans autre précision. On peut encore ajouter la lampe
$\mathrm{K}$
816 , en terre gris clair: L. Hautecoeur, Musées el collections archéologiques de l'Algérie et de la Tunisie. Mussé Alaoui. Supplement (1910), p. 189 et pl. XCVII, 1 (la photographie correspond à $\mathrm{K} 816$ et non à $\mathrm{K} 815$ comme indiqué dans la publication).

Podvin, art. cit. n. 62, pp. 33 et 36 n. 11 pour la K 808 (Hautecoeur, op. cit. n. 70 , p. 189); Deneauve, op. cit. n. 69 , p. 192, n$^{\circ} 904$ et pl. LXXXII.

72 Deneauve, op. cit. n. $69, \mathrm{n}^{\circ} 584 \mathrm{et} \mathrm{pl}$. LX. Trouvée dans la nécropole des Officiales, elle porte la marque LM. RES.

Ibid., n'823 et pl. LXXVI. Elle est inscrite EX OF MAURICI.

${ }^{74}$ Sur cette lampe, voir J-L. Podvin, uLes lampes isiaques hors d'Égypten, infra, p. 370 pour la description et la bibliographic. Elle se trouvait dans un tombeat proche de la basilique de Dermech.

${ }^{75}$ Deneauve, op. cit. n. 69 , p. 217 n 1078 et pl. XCIX.

T6 Ibid., p. 215 , n 1068 et pl. XCVII.

Ibid., p. 194, n913 et pl. LXXXIII avec la marque MAURICI; idem, p. 222 $\mathrm{n}^{\circ} 1124$ et pl. CI; S 1926 au Louvre, publiée par V. Tran tam Tinh, «Isis et Sérapis se regardants, $R A(1970 / 1)$, p. 64 et fig. 21 p. 67.

to Podvin, art. cit. n. 62, pp. 33 et 36 n. 10 pour une lampe conservée à Luxembourg.

${ }_{99}$ Deneauve, op. cit. n. 69 , p. $129, \mathrm{n}^{\circ} 424$ et pl. XLVI (I ${ }^{\mathrm{rr}}$-début $\mathrm{II}^{\mathrm{e}}$ siècle); K 809, Hautecoeur, op. cit. n. 70, p. 189, de Damous el-Karita.

so Inédite. S 1927 au musée du Louvre: elle porte la marque C.HELVI.IAN.

s1 Du Coudray La Blanchère, Gauckler, op. cit., K 136 p. 162, au sanctuaire de Saturne.

${ }^{82}$ Kater-Sibbes, Sarapis monuments, n ${ }^{\circ} 770$ p. 141 et pl. 26. Elle porte la marque M OPPI SOSI. Un exemplaire du même type est publié dans le LLIMC, s.v. Sarapis, VII.2 Ib p. 504 .
Nous en avons recensé dix-sept: deux à buste de Sarapis à gauche, ${ }^{83}$ deux d'Héliosarapis, ${ }^{84}$ onze du couple divin affronté, la plupart dans un contexte funéraire ${ }^{85}$ auquel il faut peut-être ajouter le couple sur un pied de lampe ${ }^{86}$ une de la triade Harpocrate, Isis, Anubis ${ }^{87}$ et une d'Isis debout. ${ }^{88}$ On peut leur associer deux lampes de Lepcis minor d'Isis debout ${ }^{89}$ et d'un buste isiaque. ${ }^{90}$

Plus à l'intérieur, à Raqqada, une dizaine de lampes du couple divin affronté ont été retrouvées dans des sépultures de la nécropole. ${ }^{91}$

as P. Gauckler, E. Gouwet, P. Hannezo, Musées et collections archéologiques de l'Algéne et de la Tunisie. Musét de Sousse (1902), n ${ }^{\circ} 86$ p. 62. On peut ajouter une lampe figurant Sarapis, avec encadrement de lauriers, trouvée dans la catacombe d'Hermès: Mgr Leynaud, Les catacombes africaines. Sousse-Hadnumete (Alger, 1922), n²6 p. 320 (dépouillement Y. Mousset). Le bec est noirci.

* Gauckler, Gouwet, Hannezo, Musie de Sousse, n² p. 64 du IV siècle: c'est probablement la lampe signalée par J. J. V. M. Derksen, elsis and Serapis on lamps from North Africas, dans Hommages à M. J. Vemaseren, EPRO 68/1 (Leiden, 1978), p. 302 et pl. XLVI; A. F. Leynaud, L. Carton, «Les catacombes d'Hadrumète: première campagne de fouilless, Bulletin de la Saciété Archéologique de Sousse (1905/1), p. 106, pour une lampe sans doute tardive avec inscription néo-punique.

${ }_{85}$ Gauckler, Gouwet, Hannezo, Musée de Sousse, n $\mathrm{n}^{\circ} 17$ p. 59, avec la marque CORAS; Cdt de la Comble et Lt Hannezo, «Fouilles exécutées dans la nécropole romaine d'Hadrumèten, $B C T H(1889)$, pour quatre lampes, respectivement trouvées dans un vase au pied d'un squelette (p. 117), dans la maçonnerie entre deux sépultures (p. 121), dans une urne cinéraire (p. 124) et dans un hypogée (p. 130); BSAS (1907/2), pp. 203-205 pour une lampe de la collection Montant; A. F. Leynaud, wes catacombes d'Hadrumète: quatrième campagne de fouilles", BSAS (1908/1), . 166 peur une fragnen, BSAS $(1909 / 2)$, p. $123, \mathrm{~K}$ - 66 pour une fragmentaire qui correspond sans doute au fragment publié par Vercoutre, "Sur la céramique romaine de Soussen, $R A$ (1884/1), p. 25 et pl. II.6. Mgr Leynaud, qui reprend ces diffěrentes lampes dan Les catacombes africaines. Sousse-Hadrumète, passim, en évoque une dernière dans la catacombe de Sévère, $\mathrm{n}^{\circ} 2$ p. 432.

${ }^{36}$ Musée du Bardo $\mathrm{n}^{\circ}$ inv. K 484 p. 193. Hypothèse formulée par Tran tam Tinh, art. cit. n. 72, p. 64 n. 1 .

a7 Gauckler, Gouwet, Hannezo, Musée de Sousse, n 16 p. 59.

${ }^{20} \mathrm{P}$. Gauckler, $B C T H(1894)$, p. 269. La déesse tient le sistre de la main gauche et un sattribut indistincto de la droite

a9 Musée du Bardo ${ }^{\circ}$ inv. K 933: elle tiendrait de la main droite une feuille de lotus au-dessus d'un calathos.

${ }_{90}^{90}$ Gauckler, Gouwet, Hannezo, Musée de Sousse, $n^{\circ} 18$ p. 59. Signalons en outre une petite intaille de jaspe rouge présentant une tête très fine de Jupiter Sarapis, provenant de Sousse: A. T. Vercoutre, BSAS (1903/2), p. 161, ainsi qu'un anneau d’or très épais, simplement doré et accosté d'un petit buste de Sarapis, trouvé à Monastir: $B C T H(1894), \mathrm{n}^{\circ} 33$ pp. $270-271$.

${ }_{91}$ A. Ennabli, dans A. Ennabli, J. W. Salomonson, A. Mahjoubi, La nécropole romaine de Raqgada (Tunis, 1970-3), mais nous n'avons pu consulter cet ouvrage. II s'agirait des $\mathrm{n}^{\circ} 44,77,151,154,180,260,419,432,400,389$, et 420 . La ${ }^{\circ} 400$ porte la marque EX OFICINA C.V.S AB AQUAS REGIAS. Ces luminaires peuvent être datés de la période fin $\mathrm{II}^{e}-\mathrm{III}^{e}$ siècles. 
À Thysdrus, on a mis au jour une lampe d'Harpocrate, ${ }^{92}$ six d'Isis et Sarapis affronté ${ }^{93}$ et deux anses plastiques de Sarapis trônant, ${ }^{94}$ à El-Aouja, trois luminaires à buste de Sarapis à gauche ${ }^{95}$ et deux du couple divin. ${ }^{96}$

Une lampe de Sarapis debout $\left(\mathrm{II}^{\mathrm{e}}-\mathrm{III}^{\mathrm{e}}\right.$ siècles) émane de la région de Sfax, ${ }^{97}$ ainsi qu'une, fragmentaire, de la triade Harpocrate, Isis et Anubis, ${ }^{98}$ et six d'Isis et Héliosarapis se regardant. ${ }^{99}$ De Thaenae proviennent deux lampes déjà évoquées. ${ }^{100}$

De la même époque, huit exemplaires ont été trouvés à Theveste portant le buste de Sarapis à gauche, ${ }^{101}$ le buste d'Isis ${ }^{102}$ ceux des deux dieux conjugués, ${ }^{103}$ d'Héliosarapis et Isis affrontés ${ }^{104}$ ou Harpocrate. ${ }^{105}$

$\mathrm{Du} \mathrm{III}^{e}$, on relève des lampes de Sarapis en buste à gauche à Saf-

${ }_{92}$ Musée du Bardo $\mathrm{n}^{\circ}$ inv. K 1916, p. 202. À volutes, elle porte la marque C.OPPI.RES et peut-être datée du début de l'Empire.

${ }_{93}$ Musée du Bardo $n^{\circ}$ inv. K 1921, p. 202. Grâce à A. Merlin, «Supplément au catalogue de lampes du musće Alaoui $\left(2^{\circ}\right.$ série)m, Recue tunisienne (1915), $\mathrm{n}^{\circ} 64$ p. 324 , on apprend que deux exemplaires similaires portent la marque EX OFICINA C.V.S AB AQUAS REGIAS. Les quatre autres sont les K 813, 814, 817 et 815 , cette dernière portant encore les lettres///VS: Hautecoeur, op. cit. n. 70 , et 815 , cette dernière portant encore les lettres///VS: Hautecoeur, op. cit. n. 70,
p. 189 . p. 189.

94 R. Cagnat, «Rapport sur une mission en Tunisien, Archives des missions scientifiques et littéraires, t. IX (1882), pp. 155-156 et pl. VII. L'auteur y voit des statuettes de Hadès: elles sont tout à fait conformes au premier type de Sarapis trônant défin par V. Tran tam Tinh et M.-O. Jentel, Corpus, pp. 59-60, avec dossier évasé ver le haut. Les deux portent des traits horizontaux et verticaux à l'arrière, comme sì on avait voulu imiter maladroitement des hiéroglyphes. Cagnat signale le même motif de Sarapis trônant «sur une lampe de terre rouge trouvée également à Elmotif de Sarapis trônant «sur une
Djem», probablement sur le disque.

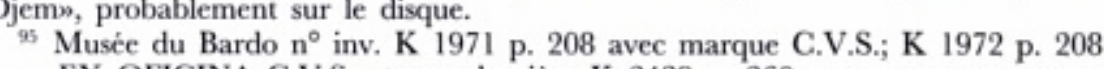
avec EX OFICINA C.V.S. et une dernière K 2429 p. 260.

${ }^{9}$ Musée du Bardo ${ }^{\circ}$ inv. K 1919 et 1920 p. 202, portant la marque INCLITI

97 V. Tran tam Tinh, Sérapis debout, ÉPRO 94 (Leiden, 1983), nIA12, p. 94.

se Massigli, op. cit. n. 30, n$^{\circ} 36$ p. 32.

9 Ibid., n ${ }^{\circ} 90$ p. 35 pour la première portant au revers la marque EX OFICINA C.V.C. AB AQUAS RE[///]AS, n²248 (1), 249 (3) et 250 (1) p. 44 pour les cinq autres qui se diffẽrencient par la couleur de la terre et le pourtour.

${ }^{100}$ Cf. supra n. 24.

101 M. Besnier, P. Blanchet, Collection du commandant Farges, n ${ }^{\circ} 173$ p. 31. Une deuxième du même type mais dont la localisation est incertaine, en terre grise, elle aussi, porte la marque L POMPO.

${ }^{102}$ Bussière, Lampes antiques d'Algérie, pp. 170 et $352, \mathrm{n}^{\circ} 3204$

${ }^{103}$ Ibid., pp. 170 et $362, n^{\circ} 3532$ et pl. 98.

104 Besnier, Blanchet, op. cit. n. $101, \mathrm{n}^{\circ} 175$ p. 32 , de terre rouge avec marque aux cinq palmes pour la première, Bussière, op. cit., pp. $169 \mathrm{et} 351-352, \mathrm{n}^{\circ} 3201-3203$ et pl. 87 pour les trois autres.

ios Besnier, Blanchet, op. cit. n. 101 , n ${ }^{\circ} 176$ p. 32 , de terre grise avec encadrement d'oves.
Saf ${ }^{106}$ et Gouraia. ${ }^{107}$ À El-Hassiba, une lampe du couple affronté ${ }^{108}$ et à Henchir es-Srira deux de Sarapis en buste à gauche ${ }^{109}$ et une de Sarapis de face. ${ }^{110}$

De Tunisie mais sans davantage de précision, on peut encore signaler quatre lampes de Sarapis en buste à gauche, ${ }^{111}$ une d'Héliosarapis de face, ${ }^{112}$ trois au moins du couple divin affronté ${ }^{113}$ et une autre du même couple vu de face, ${ }^{114}$ un buste isiaque, ${ }^{115}$ un Anubis, ${ }^{116}$ une triade, ${ }^{117}$ deux Harpocrate ${ }^{118}$ et une anse delta portant le buste d'Isis. ${ }^{119}$

À Augarmi, c'est une lampe bilychne tardive, sans doute du IV ${ }^{e}$ siècle, qui a été trouvée dans les thermes. ${ }^{120}$ Elle présente les bustes affrontés d'Isis et Héliosarapis sur piédouche, un autel entre eux, deux palmes horizontales séparant les dieux. Une lampe comparable est connue à Houareb, avec en plus une tête de lion comme anse. ${ }^{121}$ De ce dernier site provient une coupe en sigillée sur laquelle

${ }^{106}$ Ibid., $\mathrm{n}^{\circ} 291$ p. 39 , en terre rouge et encadrement de raisin et de feuilles de vigne.

${ }^{107}$ Ibid., n ${ }^{\circ} 290$ p. 39 , en terre rouge et marque $\mathrm{S}$ T(renversé) et A

1ns Tran tam Tinh, $R A(1970 / 1)$, p. 64 et fig. 20 p. 67 , a bec en coeur, grappes et feuilles de vigne sur le bandeau.

${ }^{109}$ Musée du Bardo ${ }^{\circ}$ inv. K 1318 et 1319, p. 233, à queue forcee.

${ }^{110}$ Musće du Bardo ${ }^{\circ}$ inv. K 1320, p. 233.

11 Musée du Bardo n० inv, K 2428 avec inscription OVA[////LVI, 2430 et 2431: A. Merlin, R. Lantier, Catalogue du musée Alaoui, $2^{\circ}$ supplément, p. 260; Besnier, Blanchet, op. at. n. 101, n 174 p. 32, avec marque L POMPO.

${ }_{12} \mathrm{~J}$. Brants, Antieke Terra-Cotta Lampen wit het Rijksmuseum van Oudheden te Leiden (Leiden, 1913), n77, p. 43 et pl. VI.

${ }^{113}$ Tran tam Tinh, $R A(1970 / 1)$, pp. 55-57 et 63-44, pour les lampes A et B, ainsi que celle de la collection Houdard.

114 Musée du Bardo ${ }^{\circ}$ inv. K 1917, ả rapprocher probablement de Brants, op. cit. n. 112, n895, p. 49 et pl. VI, et D. Bernal Casasola, J. del Hoyo, J. M. Pérez Rivera, "Isis en Mauretania Tingitana: Un nuevo testimonio epigrafico de su culto procedente de Septem Fratress, dans L'Africa Romana XII/3 (Sassari, 1998), pp. $1153-1154$ et fig. 6.

${ }^{115}$ Musée du Bardo ${ }^{\circ}$ inv. K 812.

${ }^{16}$ Musée du Bardo ${ }^{\circ}$ inv, K 807.

$17 \mathrm{~J}$-L. Podvin, «Sur trois lampes à huile romaines à décor égyptien du musée Sandelin (Saint-Omer'm, Bulletin trimestriel de la Sociáté Académigue des Antiquaires de la Morinie, 450 livraison, t. XXIII (juin 1992), pp. 416-417, fig. p. 422.

tt Brants, op. cit. n. $112, n^{\circ} 437$ p. 29 et pl. IV, n'523 p. 35 et pl. V.

119 Cagnat, op. cit. n. 94, pp. 156-157. La déesse porte le basileion.

${ }^{120}$ Merlin, Lantier, op. cit. n. $111, \mathrm{~K} 1918$, p. 202. A. Merlin précise dans le $B C T H$ (1915), p. CCXV, que les deux becs ont servi et que l'anse est brisée. Le pourtour est décorć de feuilles, et le revers porte la marque IAR, peut-être incomplète. ${ }^{121}$ Budde, op, cit. n. 68 , pp. 630-642, reprise par Derksen, art. cit. n. 84 , pp. 296-304. Cette lampe porte la marque EX O LARIETUS. 
sont représentés trois personnages. L'un d'eux, couronné du calathos, pourrait être Sérapis. ${ }^{122}$

Non loin d'Augarmi, le site de Gigthis a livré une lampe naviforme ${ }^{123}$ sur laquelle on peut distinguer le buste de Sarapis et la tête d'Isis, et le fort de Tisavar une lampe figurant Sarapis-Hélios. ${ }^{124}$

Également tardives sont les lampes découvertes à Haidra, présentant l'une Isis en buste à droite, ${ }^{125}$ et l'autre Sarapis en buste à gauche ${ }^{126}$ à Djilma pour Héliosarapis en buste ${ }^{127}$ ou encore à Sufetula pour Isis en buste. ${ }^{128}$

En l'état actuel de nos recensements, c'est près de cent soixante de ces lampes à thème isiaque que l'on a sorties du sol de l'actuelle Tunisie. ${ }^{129}$ Sans entrer dans les questions de méthode développées infra dans la communication de Jean-Louis Podvin, ${ }^{130}$ il faut réaffirmer que la présence d'une lampe, comme celle d'une intaille ou d'un sceau ne sont pas suffisants pour faire du lieu de découverte

${ }^{122}$ L. Budde, "Serapis oder Joseph-Serapis?" dans Festschrift J. Fink (Köln, 1984), pp. 93-96 pour qui il ne faut pas identifier le personnage barbu, comme l'a fait pp. W. Salomonon a propos d'une coupe du Bando, avec Joseph qui, representé jeune, ne devait pas circhon jeune, ne devait pas être barbu. Pour cet auteur, il s'agirait plutôt ici d'une danse giastique liée au culte de Sérapis.

${ }^{123}$ Cf. supra p. 232. Sur cette lampe comme sur celle de Carthage, voir Podvin, Les lampes isiaques hors d'Égypten, infra, pp. 370-371 pour la description et la bibliographie.

${ }^{124}$ Musée du Bardo ${ }^{\circ}$ inv. K 873: on ne sait pas si le dieu est de face ou de profil. Tisanar est un fort du limes de Tripolitaine, aujourd'hui El Hagueuff ou Ksar Rhilane, construit a l'époque de Commode (CII VIII, 11048) et utilisé par lo II limes tripolitanus (Paris, 1974), pp. 92-94; Y. Le Bohec, Les unités auxiliaires de l'amée romaine en Afrique Proconsulaire et Numidie (Paris-Aix-en-Provence, 1989), pp. 67-70.

${ }^{125}$ Besnier, Blanchet, op. cit. n. 101, n $^{\circ} 292$ p. 39 , en terre rouge. Le pourtour se compose de six feuilles lancéolées comme pour celles de Sbeitla ou de Sabratha.

${ }^{126} \mathrm{Ch}$. Lyon-Caen, Catalogue des lampes en lerre cuite grecques et chrétiennes. Musée du Louvre, n²8 pp. 97-98, CA 2703. Référence signalée par Yohann Mousset que nous remercions.

127 Derksen, art. cit. n. 84, pp. 301-302, pl. XLV et non XLIV comme indiqué par erreur p. 301

${ }^{128}$ Ibid., p. 301 et pl. XLIII. Une autre lampe de Tunisie centrale, de la fin du III $^{e}$ s., figure Isis en buste à droite. Le pourtour alterne feuilles et grappes: A. Ennabli, De Carthage à Kairouan (Paris, 1982), n²15 p. 152 ,

${ }^{129}$ La liste des sites où l'on a retrouvé de telles lampes est impressionnante: Augarmi, Bulla Regia, Calama, Carthage, Djilma, El-Aouja, El-Hassiba, Gigthis, Hadrumète, Henchir es Srira, Hitho Dianhytus, Hitpo Rhegius, Houareb, Suftulo Thenes, Thysdus, Tisavar, Tunis, sans oublier celle de Cholas, ni comper celles dont la provenance précise n'est pas connue.

Cf. infra pp. 371-379. un foyer isiaque, en gardant au mot foyer sa polysémie. Les circonstances de la présence d'un tel objet à tel endroit sont trop aléatoires pour étayer toute déduction, sauf extrapolation.

Cependant, le nombre impressionnant de ces lampes ne peut laisser indifférent même si en proportion de la quantité de lampes produites et de la variété des thèmes conçus, les lampes isiaques apparaissent comme secondaires dans toute cette production. On ne peut non plus déduire de la découverte de plusieurs lampes isiaques à proximité d'un sanctuaire consacré à nos divinités qu'un atelier fonctionnait à cet endroit, et qu'il avait vocation à créer ces objets pour la clientèle de celui-ci. On devrait également douter de la spécialisation unique de ces ateliers, en considérant que seule une production suffisamment large et variée, s'adressant à une vaste clientèle aurait permis d'assurer le bon fonctionnement de l'atelier sur le moyen ou le long terme. Or on se doit de constater que certains d'entre eux s'étaient fait une telle spécialité même si elle n'était sans doute pas unique C'est notamment le cas de l'atelier CHELIAN, pour les lampes d'Isis et d'Anubis, ${ }^{131}$ que l'on connaît seulement pour quelques autres lampes non isiaques, ou encore de EX OFICINA C.V.S.AB AQUAS REGIAS, que nous avons rencontré sur plusieurs lampes d'Isis et Héliosarapis affrontés, ${ }^{132}$ voire d'autres encore. Il faudra alors tenter de savoir sur quelle durée cette quasi mono-production a pu s'effectuer. Ceci étant, il demeure un fait, celui de l'existence d'une clientèle isiaque en Proconsulaire, clientèle de fidèles ou clientèle de circonstance lorsqu'il s'agissait de prendre part aux célébrations du 5 mars.

Pour l'intérieur de la province sont signalés différents documents anépigraphes: une stèle de marbre avec Sarapis debout ${ }^{133}$ et une base avec Isis et Anubis ${ }^{134}$ à Thugga, des statuettes de Sarapis et une statue d'Isis à Thysdrus, ${ }^{135}$ une statue en marbre blanc de Sarapis à

191 Podvin, art. ait. n. 62, pp. 34 et 36 n. 23.

15: Deux de Sfax, une de Thysdrus, une de Raggada et une de Sarapis à El-Aouja. Ulrich Hübinger, Die antiken Lampen des akademischen Kinstmuseums der Universität Bonn (Berlin, 1993), nn 244-245 p. 128 et pl. 30, signale deux exemplaires du couple Isis-Héliosarapis portant la même marque mais de provenance inconnue.

18s Tran tam Tinh, Sérapis debout, n'LA 3, fig. 3.

19 Signalée par Ch. Pícard, «L'Anubis alexandrin du Musće d'Algen», dans Studi in onore di Anistide Calderini e Roberto Paribeni, vol. III (Milano, 1956), p. 180.

is Cf. Bricault, Atlas, p. 85 et Eingartner, Isis und ihre Dienerinnen, n ${ }^{\circ} 29$ p. 120 pl. XXII pour la statue d'Isis, qu'il date de 200-210 apr. J.-C. 
Thuburbo Maius, ${ }^{136}$ une tête d'enfant avec la mèche d'Horus à Jebeniana, un buste de Sarapis en marbre à Mactaris, une plaque de calcaire avec le buste de Sarapis en relief à Ghardimaou, ${ }^{137}$ un relief isiaque avec Isis, Sarapis, Dionysos et Harpocrate à Henchir el Attermine, enfin une stèle de Morsott, dans la région de Theveste, qui présente au registre supérieur deux grandes divinités célestes - dont Saturneet au registre inférieur quatre divinités dont ne subsistent que les têtes et leurs couronnes - deux portent le basileion, une autre est cornue, la dernière diadémée-, ce qui les identifie à Isis et à d'autres divinités de son cercle. ${ }^{139}$

Tous ces documents sont à dater du $\mathrm{II}^{e}$ ou du $\mathrm{III}^{\mathrm{e}}$ siècle apr. J.-C., flonit des cultes isiaques sous l'Empire. De la même époque datent de rares documents épigraphiques: la dédicace d'une statue de Sarapis d'un prix de 3000 sesterces à Henchir Debbik ${ }^{140}$ entre 185 et 192 apr. J.-C., une simple dédicace à Sarapis de Theveste du II $^{e}$ siècle $^{141}$ de la part de deux esclaves impériaux. On ne retiendra pas en revanche deux inscriptions d'Abitina où le nom de Sarapis est restitué. $^{142}$

Ultime document indirectement lié aux cultes isiaques, une très belle mosaïque du $\mathrm{III}^{e}$ siècle découverte à Thysdrus et représentant un calendrier. Pour Novembre, on reconnait un personnage portant le masque d'Anubis, accompagné de deux ptérophores. ${ }^{143}$ Cette illus-

136 Ibid. p. 85; Yacoub, Musée du Bardo, p. 33, d'après Merlin, Musée Alaoui, suppl. 2, C 1353, p. 58. Il n'en reste que le buste qui mesure près d'un mètre de hauteur.

${ }^{137}$ Ibid., p. 85; Yacoub, Musée du Bardo, p. 32, d'après Du Coudray La Blanchère, P. Gauckler, Musée Alaoui, C 863, p. 72. Yacoub signale une stèle similaire, de provenance incertaine: Musće du Bardo $\mathrm{n}^{\circ}$ inv. 3510

${ }^{38}$ Ibid., p. 85; cf. Noèl Duval et François Baratte, aLe relief isiaque d'Henchir el Atterminen, RLowe (décembre 1982/5-6), pp. 327-334 et Eingartner, Isis und ihre Dienerinnen, ${ }^{\circ} 16$ p. 115 et pl. XV, qui le date du milieu du $\Pi^{*}$ siècle apr. J-C. 139 Cf. M. Leglay, Satume africain, I. Monuments, pp. 358-359 n56 et II. Histoire, pp. 246-247, BEFAR 205 (Paris-Rome, 1961 et 1966).

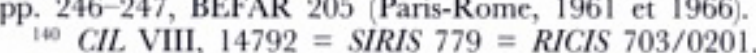

140 CIL VIII, $14792=$ SIRIS $779=$ RICIS $703 / 0201$
141 CIL VIII, $1844=$ SIRIS $782=$ RICIS $703 / 0501$.

141 CIL VIII, $1844=$ SIRIS $782=$ RICIS $703 / 0501$.
142 CIL VIII, $25842=$ SIRIS 780 pour la première.

112 CIL VIII, $25842=$ SIRIS 780 pour la première. La restitution, due à Gsell, art. cit. n. 39, p. 152 n. 9 est acceptée par Vidman; elle ne s'impose pas; of. RICIS *703/0301

CIL VIII, 25843 = SIRIS 781 pour la seconde; cf. H. G. Pflaum, eInscriptions impériales de Silan, AntAfr 3 (1969), p. 144 (= Scripta varia I p. 299). Merlin, Vidman

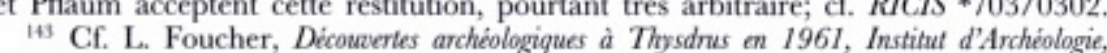

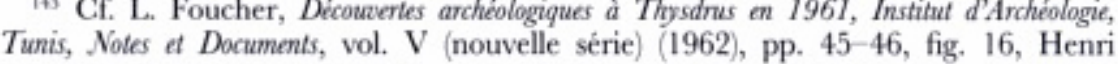

tration, que l'on retrouve ailleurs, fait référence aux Isia, qui s'étalaient sur sept jours. Commencées le 28 octobre dans la douleur de la perte d'Osiris, ces fêtes s'achevaient le 3 novembre par les Hilaria (appelées aussi Heuresis dans les Ménologes rustiques) célébrant la résurrection du dieu.

Puis les documents postérieurs à la fin du $\mathrm{III}^{e}$ siècle se font rares, hormis quelques lampes de terre cuite encore fabriquées au cours du siècle suivant. On ne sait quand les sanctuaires isiaques de Byzacène et de Zeugitane furent désertés. Ceux de Lepcis Magna et de Sabratha semblent avoir été abandonnés au cours du $\mathrm{IV}^{e}$ siècle.

Stern, «L'image du mois d'octobre sur une mosaïque d'El-Djemw, j $\mathrm{S}$ av 1965, pp 118-119 fig. 3, et id., aUn calendrier romain illustré de Thysdrus (Tunisich, Ouademo

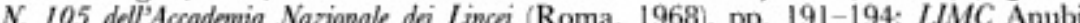
$\mathrm{n}^{\circ} 44$; voir en dernier lieu L. Bricault, «Les Anubophoresm, BSEG $24(2000 / 2001)$ pp. $40-41$ et fig. 5 . 